\title{
Analysis of Lattice Size, Energy Density and Denaturation for a One-Dimensional DNA Model
}

\author{
Gabriel Gouvea Slade, Natalia Favaro Ribeiro, Elso Drigo Filho, Jose Roberto Ruggiero \\ Department of Physics, Sao Paulo State University, São José do Rio Preto, Brazil \\ Email: ggslade@hotmail.com
}

Received January 21, 2012; revised February 30, 2012; accepted March 7, 2012

\begin{abstract}
There are several mechanical models to describe the DNA phenomenology. In this work the DNA denaturation is studied under thermodynamical and dynamical point of view using the well known Peyrard-Bishop model. The thermodynamics analysis using the transfer integral operator method is briefly reviewed. In particular, the lattice size is discussed and a conjecture about the minimum energy to denaturation is proposed. In terms of the dynamical aspects of the model, the equations of motion for the system are integrated and the results determine the energy density where the denaturation occurs. The behavior of the lattice near the phase transition is analyzed. The relation between the thermodynamical and dynamical results is discussed.
\end{abstract}

Keywords: DNA Mechanical Model; Peyrard-Bishop Model; Lattice Size; Thermal Denaturation

\section{Introduction}

The DNA molecule contains the genetic information and it is responsible for the transmission of hereditariness [1]. Since the discovery of its double helix structure done by Watson and Crick [2], researchers of several areas of science concentrated their attention to understand structural and functional aspects of this complex molecule. In the transcription and replication phenomena of DNA the ribbons separation in the double helix is an important effect, because it is necessary to expose the nitrogen bases to the solution. This means that large amplitudes and highly located motions are necessary and the dynamic of the molecule should be nonlinear.

Several models have been proposed to describe the DNA [3]. One of these is the Peyrard-Bishop (PB) model, proposed at 1989 to study DNA denaturation using statistical mechanics [4]. The original model consists of a pair of one dimensional lattice of harmonic oscillators; the adjacent oscillators of each lattice are bonded by a nonlinear Morse potential mimicking the hydrogen bond of the real molecule. The main feature of this model is to describe the separation of the double strand in terms of the average stretching of the base pairs.

The PB model and some variations [5-7] have been used to study the dynamics [8-10] and the thermodynamics [11-13] of DNA. From the dynamical point of view, there are studies about localized energy modes [14] that were identified as precursors of the denaturation and transcription process. These modes could also prescribe the dynamic of DNA in room temperature, in which large amplitude and highly localized motions had been experimentally verified $[15,16]$. From the thermodynamical point of view, the original model describes quantitatively the expected results for the DNA denaturation temperature. Recently, it has been discussed modifications in the original model in order to include a more abrupt phase transition [17].

In this work, we analyze the thermodynamical and dynamical aspects of the PB model and relate these two approaches. In the literature the study of nonlinear lattices is done for a different number of oscillators $[8,18$, 19]. Besides that, other works discuss the possibility of phase transition to finite lattices $[20,21]$. The main objective of the paper is to suggest a criterion to fix the minimum size of the lattice. In order to get this result, we use the transfer integral operator method [22] and calculate the error committed in the partition function of the system. Thermodynamic results also lead to conjecture a minimum energy per base pair for occurrence of the lattice phase transition. This threshold energy can also be verified from dynamical results. We propose that the phase transition can be dynamically observed by following the time evolution of the position of the oscillators for different energies.

\section{The Peyrard-Bishop Model}

In the Peyrard-Bishop original model each strand of the macromolecule is represented by a harmonic lattice and 
the interaction between them is described by a nonlinear potential. The three-dimensional aspects of the helicoidally structure are not considered and only the motions perpendicular to the strands are analyzed [4]. The nucleotides positions are denoted by $u_{j}$ and $v_{j}$, respectively, with $j=1,2, \cdots, N$. For simplicity, we assume that the chains are homogeneous, i.e., all masses and all elastics constants are equal.

The Hamiltonian of the model for a homogeneous chain is expressed as:

$$
\begin{aligned}
H_{P B}=\sum_{j=1}^{N} & \left\{\frac{m}{2}\left(\dot{u}_{j}^{2}+\dot{v}_{j}^{2}\right)+\frac{k}{2}\left(u_{j+1}-u_{j}\right)^{2}\right. \\
& \left.+\frac{k}{2}\left(v_{j+1}-v_{j}\right)^{2}+D\left[\exp \left(-a\left(u_{j}-v_{j}\right)\right)-1\right]^{2}\right\}
\end{aligned}
$$

The last term in Equation (1) correspond to the Morse potential used to describe the hydrogen bonds, the parameters $D$ and $a$ are related, respectively, with the depth and width of the potential well, $k$ is the elastic constant of the harmonic potential used to simulate the stacking interaction and $m$ is the mass. The Hamiltonian (1) can be uncoupled by introducing new variables $x_{j}=\frac{\left(u_{j}+v_{j}\right)}{\sqrt{2}}$ and $y_{j}=\frac{\left(u_{j}-v_{j}\right)}{\sqrt{2}}$. The $H_{P B}$ Hamiltonian can be written as the sum of two terms, one of them depending only on $x$ variable representing a chain of harmonic oscillators and it is not important for our analysis here. On the other hand, the Hamiltonian dependent on $y$ variable can be written as

$$
\begin{aligned}
H_{y}=\sum_{j=1}^{N} & \left\{\frac{m}{2} \dot{y}_{j}^{2}+\frac{k}{2}\left(y_{j+1}-y_{j}\right)^{2}\right. \\
& \left.+D\left[\exp \left(-a \sqrt{2} y_{j}\right)-1\right]^{2}\right\}
\end{aligned}
$$

This Hamiltonian represents a chain of harmonic oscillators with the additional on site Morse potential that carries the nonlinearity of the model.

\section{Thermodynamical Analysis}

The thermodynamic analysis of the system is described by the partition function $Z$ expressed in terms of the variable representing the average stretching of the base pairs $\left(y_{n}\right)$, i.e.,

$$
\begin{aligned}
Z & =\int \delta y \exp (-\beta H(y)) \\
& =\int \prod_{n=1}^{N} \mathrm{~d} y_{n} \exp \left(-\beta H\left(y_{n}, y_{n-1}\right)\right)
\end{aligned}
$$

where $N$ is the number of base pairs in the chain, $\beta=\frac{1}{k_{B} T}, k_{B}$ is the Boltzmann constant, $T$ is the tem- perature and $H\left(y_{n}, y_{n-1}\right)$ is the uncoupled Hamiltonian of the model, given by Equation (2).

The transfer integral operator method [22] is used to determine the thermodynamical properties from the partition function. This method allows relating the partition function with eigenfunctions $\psi_{n}$ and eigenvalues $\varepsilon_{n}$ given by the following pseudo-Schrödinger equation:

$$
\begin{aligned}
& {\left[-\frac{1}{2 \beta^{2} k} \frac{\mathrm{d}^{2}}{\mathrm{~d} y_{i}^{2}}+V\left(y_{i}\right)\right] \psi_{n}\left(y_{i}\right)} \\
& =\left[\varepsilon_{n}+\frac{1}{2 \beta} \ln \left(\frac{2 \pi}{\beta k}\right)\right] \psi_{n}\left(y_{i}\right)
\end{aligned}
$$

where $V\left(y_{i}\right)$ is the Morse potential. From the solutions of Equation (4), the partition function can be obtained by the sum $Z=\sum_{n} \exp \left(-N \beta \varepsilon_{n}\right)$. However, in the thermodynamical limit in which the number of particles is very large $(N \rightarrow \infty)$, the partition function is dominated by the ground state. For this reason, the analysis of the problem is usually limited to determine the energy eigenfunction and eigenvalue for the ground state.

The characterization of the phase transition for DNA molecule is done following the dependence of an order parameter with the temperature of the system. Usually, the order parameter is the average stretching of base pairs $(<y>)[4,11]$.

The eigenfunctions that are solution of the pseudoSchrödinger Equation (4) can be characterized as a probability density. In this way, the average stretching of the base pairs can be obtained from the equality:

$$
\langle y\rangle=\frac{\int_{-\infty}^{\infty} y \psi^{2} \mathrm{~d} y}{\int_{-\infty}^{\infty} \psi^{2} \mathrm{~d} y}
$$

There are several methods that can be used to determine the solutions of Equation (4), for example, the Laplace transforms [23] and by using the factorization method [24]. Then, the eigenenergy and eigenfunction for the ground state of Morse potential are known (see, for example, references $[4,11])$ :

$$
\begin{aligned}
& \psi_{0 M}=(\sqrt{2} a)^{1 / 2} \frac{(2 d)^{d-1 / 2}}{[\Gamma(2 d-1)]^{1 / 2}} \exp \left(-d \mathrm{e}^{-\sqrt{2} a y}\right) \\
& \exp \left[-\left(d-\frac{1}{2}\right) \sqrt{2} a y\right]
\end{aligned}
$$

and

$$
\varepsilon_{0 M}=\frac{1}{2 \beta} \ln \left(\frac{\beta k}{2 \pi}\right)+\frac{a}{\beta}\left(\frac{D}{k}\right)^{1 / 2}-\frac{a^{2}}{4 \beta^{2} k}
$$


where the condition $d \equiv\left(\frac{\beta}{a}\right)(k D)^{1 / 2}>\frac{1}{2}$ must be observed in order to exist bond states.

From the ground state eigenfunction given by (6), the average stretching of base pairs can be found by using Equation (5). To this end it is necessary to fix the parameters which characterize the model. Following the reference [25], the better parameters for Morse potential to adjust to DNA are $D=0.03 \mathrm{eV}$ and $a=2.81 \AA^{-1}$ and the elastic constant to the harmonic potential is $k=0.06$ $e V \cdot \AA^{-2}$. Using these parameters, the behavior of $\langle y>$ with temperature has an increase in the average stretching of the base pairs, indicating the thermal denaturation of the DNA molecule in the temperature range from 300 to 350 $\mathrm{K}$.

Other approach to determine the melting temperature can be made by noting that the eigenfunction obtained from Equation (4) must be quadratically integrable. Then, it is possible to determine from the eigenfunction (6) a critical temperature of the phase transition $\left(T_{C}\right)$. The eigenfunction (6) is quadratically integrable only if $d$ is higher than 0.5 . For $d$ lower or equal 0.5 the system does not have a discrete bond states and the square of the wave function integrated in all space diverges. Then, the critical temperature $T_{C}$ is obtained from $d=0.5$ and it is given by

$$
T_{C}=\frac{2 \sqrt{k D}}{a k_{B}}
$$

Substituting the parameters $D, a, k$ and the Boltzmann constant, $k_{B}=8.617 \times 10^{-5} \mathrm{eV} / \mathrm{K}$, in Equation (8) the value of the critical temperature is, approximately, $350 \mathrm{~K}$. The reference [1] indicates that the denaturation temperature of the DNA molecule varies in the range 318 to $372 \mathrm{~K}$, depending on the nucleotides composition of the chain. In this way, the obtained critical temperature is consistent with experimental results.

The thermodynamical treatment of the Peyrard-Bishop model to DNA was realized assuming a very large number of oscillators in the chain. In this limit, the excited states to the Morse potential were disregard. If the number of oscillators is not large, it is necessary to estimate the numerical error in this approach. The partition function (3), written in terms of the eigenvalues of Equation (4), can be rearranged in the following form:

$$
\begin{aligned}
Z & =\sum \exp \left(-N \beta \varepsilon_{n}\right) \\
& =\exp \left(-N \beta \varepsilon_{0}\right)+\exp \left(-N \beta \varepsilon_{1}\right)+\cdots \\
& =\exp \left(-N \beta \varepsilon_{0}\right)\left[1+\exp \left(-N \beta\left(\varepsilon_{1}-\varepsilon_{0}\right)\right)+\cdots\right]
\end{aligned}
$$

Then, the first order error in the partition function is $\exp \left(-N \beta\left(\varepsilon_{1}-\varepsilon_{0}\right)\right)$. It is important to note that the series (9) must be convergent otherwise the thermodynamic limit could not be valid to none value of the particles number $N$. So the higher terms in (9) are always lower than the first one. In order to estimate the value of $N$, we should know the difference between the energy eigenvalues $\varepsilon_{1}$ and $\varepsilon_{0}$. This estimative can be done remembering that the Morse potential permits bond states to the values of $n=0,1,2, \cdots,\left(d-\frac{1}{2}\right)$, with $d=\left(\frac{\beta}{a}\right)(k D)^{1 / 2}$. Using the parameters given above and the temperature equal to $300 \mathrm{~K}$, the value obtained to $\left(d-\frac{1}{2}\right)$ is approximately 0.08 . This means that there is only one bond state inside the potential well at this temperature, the ground state. Increasing the temperature above $350 \mathrm{~K}$ even the ground state will be out of the potential well. On the other hand, a discrete first excited state appears only for temperature lower than $150 \mathrm{~K}$.

Thus, for the temperature range of interest, the first excited state is in the continuum part of the spectrum. In this case, the difference between the ground state and the first energy level of the continuum spectrum can be estimated as the value of the depth of the well, $D$, minus the value of the ground state energy, $\varepsilon_{0}$, i.e.,

$\Delta \varepsilon=\varepsilon_{1}-\varepsilon_{0} \cong D-\varepsilon_{0}$. Consequently, the error by disregarding the first state above the ground state is $\exp (-N \beta \Delta \varepsilon)$. From the used parameters and considering the size of the chain equal to 21 oscillators, the error in the partition function by disregarding the second term in the expansion (9) at $300 \mathrm{~K}$ is lower than $10^{-5}$. This result indicates that a chain with 21 oscillators can be considered as being sufficiently large to disregard the states above the ground state. This procedure can be adopted to control the error in terms of the lattice size. It is important to observe that for the system close the denaturation temperature, the eigenvalue $\varepsilon_{0}$ is close the top of the potential well, i.e., $\Delta \varepsilon$ becomes minor. Then, the continuum levels of the spectrum become more and more important to the description of the system.

Considering the above arguments, the characterization of the phase transition of the DNA molecule by the thermodynamical formalism can be done admitting that all the base pairs have sufficient energy to break the Morse potential, i.e., when the oscillators have energy above the potential well. In this way, when the energy of each base pair is equal to or higher than $D$, the wave function is no more quadratically integrable and the phase transition occurs. An alternative way to analyze this relation between the melting process and the energy of each base pair is to note that the energy density of the lattice can be related with the melting temperature by the relation $\frac{E}{N}=k_{B} T$. As the critical temperature obtained 
is $350 \mathrm{~K}$, we can predict that the energy density necessary to denaturation is $\frac{E}{N}=0.03 \mathrm{eV} /$ oscillator. One observes that this value is the same that was used to the depth of the potential well $D$ which reinforce the previous arguments. Summarizing, from this analysis, the denaturation occurs when the energy per oscillator is at least equal to the depth of the Morse potential well.

\section{Dynamical Study of the Model}

In this section the dynamical results for the PeyrardBishop model are discussed. The numerical calculation can be simplified if we introduce dimensionless variables:

$\xi_{j}=a \sqrt{2} y_{j}$ and $\tau=2\left(\frac{D a^{2}}{m}\right)^{\frac{1}{2}} t$. The Hamiltonian

can be rewritten as a function of a single parameter:

$$
H=\sum_{j=1}^{N}\left\{\frac{1}{2} \dot{\xi}_{j}^{2}+\frac{1}{2} C\left(\xi_{j+1}-\xi_{j}\right)^{2}+\frac{1}{2}\left(\exp \left(-\xi_{j}\right)-1\right)^{2}\right\}
$$

where $\dot{\xi}=\frac{\mathrm{d} \xi}{\mathrm{d} \tau}, C=\frac{k}{4 D a^{2}}$ and $H_{y} \equiv 2 D H$. So the equations of motion are given by:

$$
\ddot{\xi}_{j}=C\left(\xi_{j+1}+\xi_{j-1}-2 \xi_{j}\right)+\exp \left(-2 \xi_{j}\right)-\exp \left(-\xi_{j}\right)
$$

We integrate the equations of motion using the tenth order Runge-Kutta Nystrom method [26]. The initial condition is such that only the central oscillator of the chain is excited and all its energy is kinetic. Then, at time $t=0$ all oscillators are in their equilibrium position and at rest with the exception of the central oscillator that has an initial velocity. The periodic boundary conditions is used, i.e., $\xi_{0}=\xi_{N}$ and $\xi_{N+1}=\xi_{1}$. Following the previous discussion (Section 3), the number of oscillators used to perform the simulation is 21 .

In Section 3, we conjecture that the denaturation happens if each oscillator shares an energy corresponding to the depth of the Morse potential well, D. By using the dimensionless variables, as writing in (10), this depth is tracked back to the value 0.5 . In order to characterize the denaturation, we follow the position value $(y)$ of each oscillator and its Morse potential energy as a function of the time. We consider that the chain reaches the denaturation when all the oscillators get the Morse potential equal to or larger than 0.5 . In this case all the hydrogen bonds should be broken and the double strand should be separated. Figure 1 shows the position $y$ of all the oscillators superposed as a function of the time. In this simulation it is used $N=21$ and the energy density is 0.7857. Thus, in Figure 1 there are 21 curves superposed, there is one curve for each site of the lattice. When all the oscillators acquire energy of the Morse potential equiva-

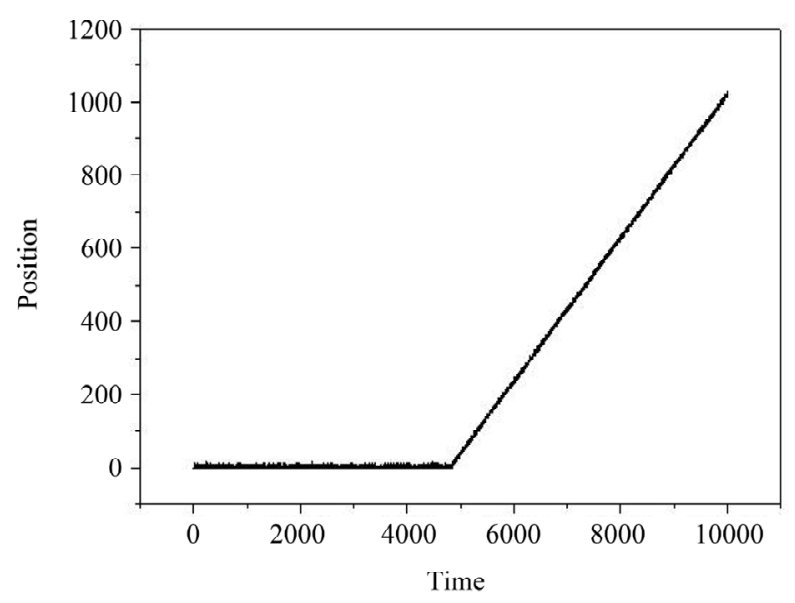

(a)

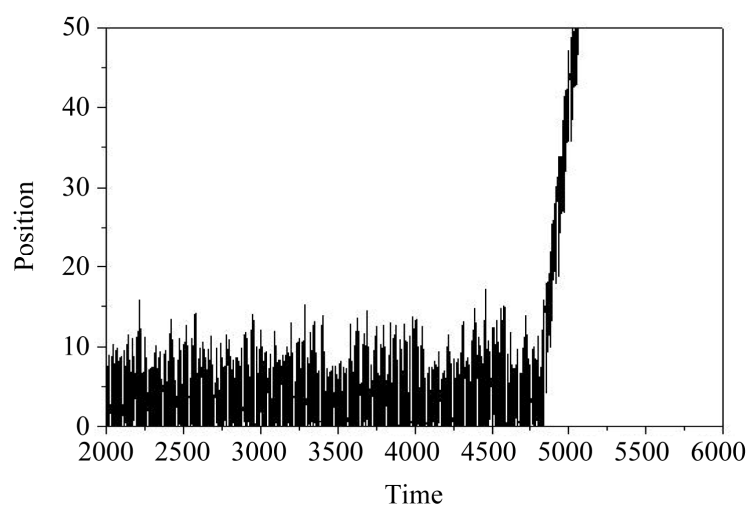

(b)

Figure 1. (a) Position of each of the oscillators superposed as a function of the time; (b) A zoon in the region where the onsite potential is broken down. $N=21$ and $\frac{E}{N}=0.7857$.

lent to the depth of the well, the positions of all oscillators begin to rise constantly, so the chain starts a uniform translation motion. Once $y$ represents the separation of a base pair, this behavior characterizes qualitatively the broken of the hydrogen bonds and consequently the DNA denaturation.

Another result obtained from the dynamics is related with the behavior of the chain before the phase transition of the lattice. Following the time evolution of the energy of Morse for each oscillator we observe the behavior of the hydrogen bond in the denaturation process. A cooperative effect in this process is observed. Specifically, it is noted that when one hydrogen bond is broken it induces a broken of some neighbour hydrogen bonds forming "bubbles" in the lattice. This occurs in different parts of the chain and these regions grow up until the complete separation of the lattices, i.e., the denaturation occurs. This behavior is shown in the Figure 2 where the temporal evolution of the hydrogen bond break of each oscillator can be observed. In this figure the site bonded is 


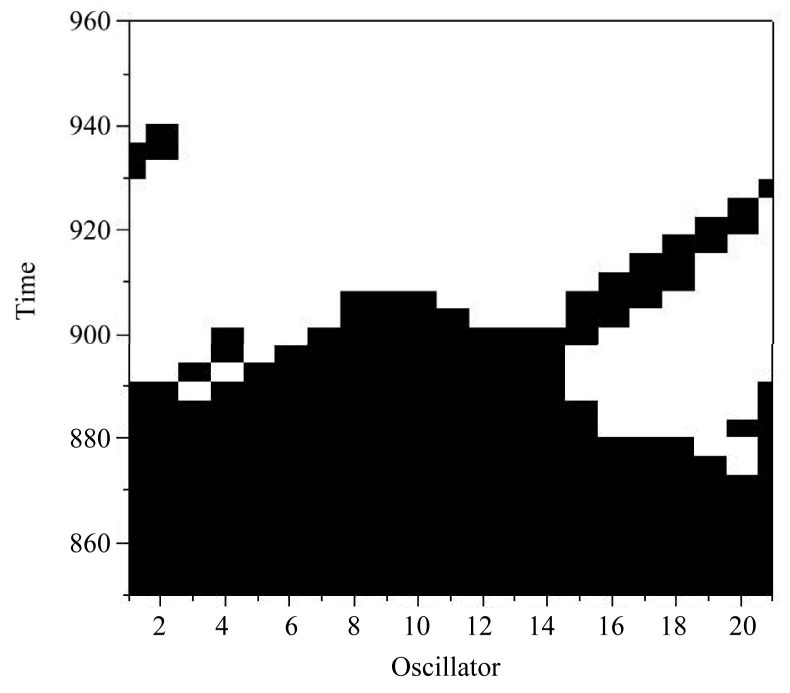

Figure 2. Hydrogen bond of each oscillator in function of the time. Black color represents the formed bonds, while white color represents the broken bond. $N=21$ and $\frac{E}{N}=\mathbf{0 . 8 8 1}$.

represented by black color and the broken hydrogen bond is indicated by the white color.

Finally we analyze the energy density in the model. Then we simulate different energy densities and verify the necessary time to denaturation. This result is presented in Figure 3. We note that small values of the energy density correspond to high values of time to obtain the denaturation. The results suggest also that an asymptotic behavior could be achieved for energy density near 0.5 , which is the well depth, in accordance with the thermodynamic results. Others initial conditions were tested and the results are qualitatively in agreement with these.

\section{Conclusions}

In this work we studied the thermodynamic and dynamic properties of the one dimensional model to study the DNA macromolecule. The thermodynamical analysis inferred that a chain of 21 oscillators should be sufficient large to analyze the behavior of the system for room temperatures $(300 \mathrm{~K})$, once that the error in the partition function is about $10^{-5}$ when despising the excited states of the Schrödinger-type Equation (4). It is important to observe that, if the system is near the denaturation temperature, the thermodynamic results indicate that it is necessary a large chain to describe its properties, otherwise the error in the partition function becomes significant.

It is suggested a dynamical approach to analyze the thermal denaturation by studying the stretching of the base pairs $(y)$ for each oscillator on the lattice. This analysis permits to observe that the formation of bubbles is

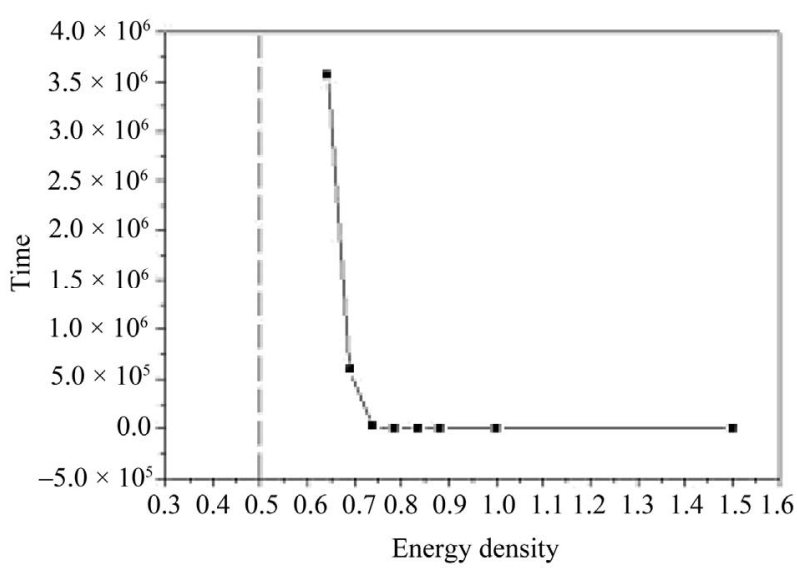

Figure 3. Energy density of the system as a function of the necessary time to occur denaturation. $N=21$.

part of the melting process. The minimum energy density needed to the denaturation is related with the energy of the potential well, i.e., it is necessary that all oscillators of the chain get energy higher than the parameter $D$ of the Morse potential to get the melting. The dynamical calculations of the model, in a lattice of 21 oscillators, show that the denaturation occurs when the energy densities tends to the value of $D$. However, for the obtained results the energy is always higher than that indicated from thermodynamics analysis. Two causes can be identified to this behavior, first the energy in the dynamic simulation are not localized only in the Morse potential, part of it can be localized in the harmonic potentials. Other cause is related with the fact that the thermodynamics results indicate that near the denaturation temperature it is necessary a larger chain to describe the system. Nevertheless the behavior of the chain is in agreement with the DNA phenomenology and both formalisms converge to the same result.

\section{Acknowledgements}

The authors are grateful to Capes and CNPq for the financial support.

\section{REFERENCES}

[1] W. Saenger, "Principles of Nucleic Acid Structure," Springer-Verlag Press, New York, 1984. doi:10.1007/978-1-4612-5190-3

[2] G. B. Watson and F. H. Crick, "Molecular Structure of Nucleic Acids-A Structure for Deoxiribose Nucleic Acid," Nature, Vol. 171, 1953, pp. 737-738. doi:10.1038/171737a0

[3] L. Yakushevich, "Nonlinear Physics of DNA," Wiley Series in Nonlinear Science, Chichester, 1998.

[4] M. Peyrard and A. R. Bishop, "Statistical Mechanics of a Nonlinear Model for DNA Denaturtion," Physical Review Letters, Vol. 62, No. 23, 1989, pp. 2755-2758. 
doi:10.1103/PhysRevLett.62.2755

[5] T. Dauxois, M. Peyrard and A. R. Bishop, "EntropyDriven DNA Denaturation," Physical Review E , Vol. 47, No. 1, 1993, pp. 44-47. doi:10.1103/PhysRevE.47.R44

[6] M. Joyeux and S. Buyukdagli, "Dynamical Model Based on Finite Stacking Enthalpies for Homogeneous and Inhomogeneous DNA Thermal Denaturation," Physical Review E, Vol. 72, No. 5, 2005, Article ID: 051902. doi:10.1103/PhysRevE.72.051902

[7] M. Peyrard, S. Cuesta-López and G. James, "Nonlinear Analysis of the Dynamics of the DNA Breathing," Journal of Biological Physics, Vol. 35, No. 1, 2009, pp. 73-89. doi:10.1007/s10867-009-9127-2

[8] E. Zamora-Sillero, A. V. Shapovalov and F. J. Esteban, "Formation, Control, and Dynamics of N Localized Structures in the Peyrard-Bishop Model," Physical Review E, Vol. 76, No. 6, 2007, Article ID: 066603. doi:10.1103/PhysRevE.76.066603

[9] S. Zdravkovic and M. V. Sataric, "DNA Dynamics and Big Viscosity," International Journal of Modern Physics B, Vol. 17, No. 31-32, 2003, pp. 5911-5923. doi:10.1142/S0217979203023513

[10] J. De Luca, E. Drigo Filho, A. Ponno and J. R. Ruggiero, "Energy Localization in the Peyrard-Bishop DNA Model," Physical Review E, Vol. 70, No. 2, 2004, Article ID: 026213. doi:10.1103/PhysRevE.70.026213

[11] M. Peyrard, "Nonlinear Dynamics and Statistical Physics of DNA," Nonlinearity, Vol. 17, No. 2, 2004, pp. 1-40. doi:10.1088/0951-7715/17/2/R01

[12] G. Weber, "Sharpe DNA Denaturation Do to Solvent Interaction," Europhysics Letters, Vol. 73, No. 5, 2006, p. 806. doi:10.1209/epl/i2005-10466-6

[13] N. F. Ribeiro and E. Drigo Filho, "Using a One-DimenSional Lattice Applied to the Thermodynamic Study of DNA," Journal of Physics: Conference Series, Vol. 246, 2010, Article ID: 012037. doi:10.1088/1742-6596/246/1/012037

[14] G. G. Slade, E. Drigo Filho and J. R. Ruggiero, "Stability of Breathres in Simple Mechanical Models for DNA," Journal of Physics: Conference Series, Vol. 246, 2010, Article ID: 012039. doi:10.1088/1742-6596/246/1/012039

[15] S. W. Englander, N. R. Kallenbach, A. J. Heeger, J. A. Krumhansl and A. Litwin, "Nature of the Open State in Long Polynucleotide Double Helix-Possibility of Soliton Excitations," Proceedings of the National Academy of Sciences, Vol. 77, No. 12, 1980, pp. 7222-7226. doi:10.1073/pnas.77.12.7222

[16] J. L. Leroy, M. Kochoyan, T. Huynh-Dinh and M.
Guéron, "Characterization of Base-Pair Opening in the Deoxynucleotide Duplexes Using Catalyzed Exchange of the Imino Proton," Journal of Molecular Biology, Vol. 200, No. 2, 1988, pp. 223-238. doi:10.1016/0022-2836(88)90236-7

[17] M. Peyrard, S. Cuesta-López and D. Angelov, "Experimental and Theoretical Studies of Sequence Effects on the Fluctuation and Melting of Short DNA Molecules," Journal of Physics: Condesed Matter, Vol. 21, No. 3, 2009, Article ID: 034103. doi:10.1088/0953-8984/21/3/034103

[18] J. M. Silva, E. Drigo Filho and J. R. Ruggiero, "Localization and Delocalization of Energy in a Peyrard-Bishop Chain," The European Physical Journal E: Soft Matter and Biological Physics, Vol. 29, No. 2, 2009, pp. 245-251. doi:10.1140/epje/i2009-10475-9

[19] B. S. Alexandrov, L. T. Wille, K. Ø. Rasmussen, A. R. Bishop and K. B. Blagoev, "Bubble Statistics and Dynamics in Double-Stranded DNA," Physical Review E, Vol. 74, No. 5, 2006, Article ID: 050901. doi:10.1103/PhysRevE.74.050901

[20] N. Theodorakopoulos, "Thermodynamic Instabilities in One-Dimensional Particle Lattices: A Finite-Size Scaling Approach," Physical Review E, Vol. 68, No. 2, 2003, Article ID: 026109. doi:10.1103/PhysRevE.68.026109

[21] S. Buyukdagli and M. Joyeux, "Theoretical Investigation of Finite Size Effects at DNA Melting," Physical Review E, Vol. 76, No. 2, 2007, Article ID: 021917. doi:10.1103/PhysRevE.76.021917

[22] D. J. Scalapino and M. Sears, "Statistical Mechanics of One-Dimensional Ginzburg-Landau Fields," Physical Review B, Vol. 6, No. 9, 1972, pp. 3409-3416. doi:10.1103/PhysRevB.6.3409

[23] G. Chen, "The Exact Solutions of the Schrodinger Equation with the Morse Potential via Laplace Transforms," Physical Review A, Vol. 326, No. 1-2, 2004, pp. 55-57. doi:10.1016/j.physleta.2004.04.029

[24] E. D. Filho and R. M. Ricotta, "Morse Potential Energy Spectra through the Variational Method and Supersymmetry," Physics Letters A, Vol. 269, No. 5-6, 2000, pp. 269-276. doi:10.1016/S0375-9601(00)00267-X

[25] R. A. S. Silva, E. D. Filho and J. R. Ruggiero, "A Model Coupling Vibrational and Rotational Motion for DNA Molecule," Journal of Biological Physics, Vol. 34, No. 5, 2008, pp. 511-519. doi:10.1007/s10867-008-9111-2

[26] C. Tsitouras, "A Tenth Order Sympletic Runge-KuttaNystrom Method," Celestial Mechanics and Dynamical Astronomy, Vol. 74, No. 4, 1999, pp. 223-230. doi:10.1023/A:1008346516048 